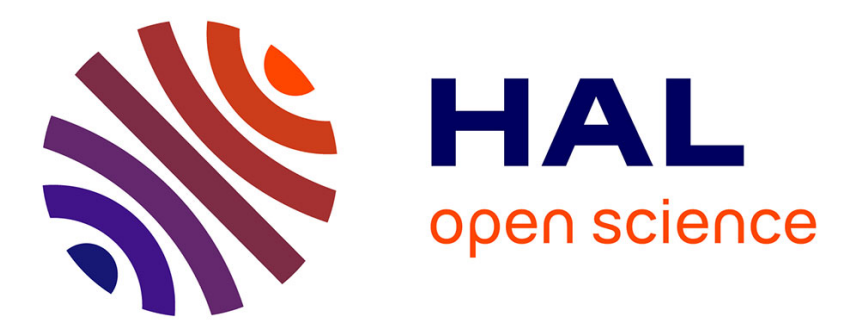

\title{
Position and velocity estimation through acceleration measurements
}

\author{
Antonio Estrada, Denis Efimov, Wilfrid Perruquetti
}

\section{To cite this version:}

Antonio Estrada, Denis Efimov, Wilfrid Perruquetti. Position and velocity estimation through acceleration measurements. 19th IFAC World Congress, Aug 2014, Cape Town, South Africa. hal-00966200

\section{HAL Id: hal-00966200 \\ https://hal.inria.fr/hal-00966200}

Submitted on 26 Mar 2014

HAL is a multi-disciplinary open access archive for the deposit and dissemination of scientific research documents, whether they are published or not. The documents may come from teaching and research institutions in France or abroad, or from public or private research centers.
L'archive ouverte pluridisciplinaire HAL, est destinée au dépôt et à la diffusion de documents scientifiques de niveau recherche, publiés ou non, émanant des établissements d'enseignement et de recherche français ou étrangers, des laboratoires publics ou privés. 


\title{
Position and velocity estimation through acceleration measurements
}

\author{
Antonio Estrada* Denis Efimov ${ }^{*, *}$ Wilfrid Perruquetti * \\ * Non-A Team, Inria Lille - Nord Europe,Parc Scientifique de la Haute \\ Borne, 40 avenue Halley, 59650 Villeneuve d'Ascq, France. \\ ** National Research University ITMO, 49 avenue Kronverkskiy, \\ 197101 Saint Petersburg, Russia. \\ e-mail addresses: xheper@yahoo.com,denis.efimov@inria.fr, \\ wilfrid.perruquetti@inria.fr
}

\begin{abstract}
This paper proposes a solution to the problem of velocity and position estimation for a class of oscillating systems whose position, velocity and acceleration are zero mean signals. The proposed scheme considers that the dynamic model of the system is unknown and only noisy acceleration measurements are available.
\end{abstract}

\section{INTRODUCTION}

The estimation of position/velocity through the integration of measurements from inertial sensors inherently causes errors to grow with time, commonly known as integration drift. In fact, the process of zero-mean noise integration leads to an output that increases with integration time, even in the case that the accelerometer is at rest Thong et al. (2004). For this reason, velocity/position estimation based on inertial sensors requires some additional source of information providing for the corresponding correct signal value at some time instants. In general this information is updated with intervals significantly larger than the acceleration measurement rate. For instance, in inertial navigation systems (INS) aiding sensors are applied. An INS consists of a triad of orthogonal accelerometers and rate gyroscopes that are used to calculate the both the translational and rotational movements of an aircraft over time Rogers (2003), a process know as dead reckoning. MEMS technology has made low-cost INS available. However, sensor biases coupled with the time integration relationships needed for dead reckoning cause that the solution quickly diverges. That is why externally referenced sensors, such as the global positioning system, are required to regulate the error growth Grewal et al. (2007); Kalman filters are commonly used in order to fuse the two sources of information.

An alternative to the use of aiding sensors is the use of prior-knowledge of motion. In Liu et al. (2009), for instance, the drift accumulation from gyroscope based orientation estimation, in human walking experiments, is compensated by detecting different gait phases with the aid of accelerometers and the knowledge of the human walking motion. In Schepers et al. (2010), foot placement for several strides is estimated. The drift is reduced applying the knowledge of the cyclic walking movement that leads to information on initial and final conditions.

\footnotetext{
1 This work was supported in part by the Government of Russian Federation (Grant 074-U01) and the Ministry of Education and Science of Russian Federation (Project 14.Z50.31.0031).
}

In the present work we address the problem of velocity/position estimation based only in inertial information with no aiding sensor nor an explicit dynamical model. We propose a method that allows to adjust the estimated position for the case in which the acceleration measurements, which can be contaminated by zero mean noise, are unbiased and correspond to an oscillating motion with zero mean velocity and position. This proposal compensates the drift using the oscillating motion period. Then, a period estimation algorithm is fundamental considering that the oscillation period is in general not available. In Spence and Clarke (2000), a method for estimating the period and amplitude profile of a signal with multiple periodicities is proposed based on the results reported in Feder (1993). In Spence and Clarke (2000), a likelihood computation is put in terms of a least mean square (LMS) residual power expression which is evaluated for different test periods. The estimated period is chosen as the one minimising the LMS residual power. In the field of music and speech analysis the pitch period (fundamental frequency) estimation is very important. For short-time estimation of pitch period, the algorithms that find the average fundamental frequency using autocorrelation or linear prediction techniques are the most commonly used Guangyu and Shize (2009), Peeters (2006), Bernardin (2006), Min et al. (2005). We propose a period estimation algorithm that takes advantage on the zero mean nature of the signals. This scheme is not restricted to sinusoidal dominant frequency and is computationally simple. Relying on the aforementioned oscillation period estimation, a solution for finite time tracking of position and velocity is proposed.

The rest of the paper is organized as follows, in the next section the problem statement is presented. In section III the possibility of an asymptotic velociy/position estimation is discussed and the idea of using the oscillation period is introduced. Section IV is devoted to the period estimation proposal. In Section $\mathrm{V}$ the scheme for finite-time position/velocity estimation is presented. Finally, Section VI presents simulation results. 


\section{PROBLEM STATEMENT}

Consider a system with the following acceleration dynamics

and measurement model

$$
\ddot{x}=f(x, \dot{x})
$$

$$
\Psi(t)=\ddot{x}(t)+\eta
$$

where $\eta$ is a bounded zero-mean noise. The acceleration measurement is assumed unbiased. $f(x, \dot{x})$ is an unknown nonlinear function. It is assumed that the position $x$, and hence $\dot{x}, \ddot{x}$, have a periodic or quasi-periodic behaviour, more precisely it is considered that position is oscillating between two bounds, (there exist two constants such that $\left.B_{m} \leq x^{(i)}(t) \leq B_{M}, \quad \forall t>0, i=0,1,2\right)$. The objective of this work is to present an alternative solution for velocity and position estimation in the case of only acceleration measurement for a class of periodical signals, i.e. $x^{(i)}(t)=$ $x^{(i)}\left(t+T_{f}\right)$ for some finite $T_{f}$.

\section{VELOCITY ESTIMATION}

\subsection{Velocity estimation through averaging}

In the following the super index $\langle i\rangle$ is used to indicate the $i$-th time integral of a function $y: R \rightarrow R$ :

$$
y^{<i>}(t)=\int_{0}^{t} \ldots \int_{0}^{\tau_{2}} y\left(\tau_{1}\right) d \tau_{1} \ldots d \tau_{i} .
$$

Consider (2), in order to obtain velocity estimation we proceed to integrate directly $\Psi(t)$, then

$$
\begin{aligned}
& \Psi^{<1>}(t)=\dot{x}(t)-\dot{x}(0)+\eta^{<1>}, \\
& \Psi^{<2>}(t)=x(t)-x(0)-\dot{x}(0) \cdot t+\eta^{<2>},
\end{aligned}
$$

dividing (5) by $t$ we obtain

$$
\frac{\Psi^{<2>}(t)}{t}=-\dot{x}(0)+\frac{x(t)-x(0)+\eta^{<2>}}{t} .
$$

Then, define the velocity estimation, $\hat{\dot{x}}$, as follows:

$$
\begin{aligned}
\hat{\dot{x}} & =\Psi^{<1>}(t)-\frac{\Psi^{<2>}(t)}{t} \\
& =\dot{x}(t)+\eta^{<1>}-\frac{x(t)-x(0)+\eta^{<2>}}{t} .
\end{aligned}
$$

Let us recall that the position is bounded and that the noise is zero-mean and bounded. Then, the next limit can be obtained

$$
\lim _{t \rightarrow \infty}\left\{\frac{x(t)-x(0)+\eta^{<2>}}{t}\right\}=0 .
$$

Therefore, for $t$ "sufficiently" large

$$
\hat{\dot{x}} \simeq \dot{x}(t)+\eta^{<1>} \text {. }
$$

The amount of time, which is necessary to obtain a reliable estimation, will depend on the magnitude of the bounds of position and noise. Next, a similar averaging approach can be applied for position estimation. Such estimation would be asymptotic without a possibility to regulate the speed of convergence. In addition, integration on an infinite time interval can be technically difficult without a significant accumulation of computation error leading to large estimation error. Thus a finite time solution has to be found. In fact a possibility to find a solution in finite time is related with knowledge of the period. In next section, this statement about the necessity of period knowledge is further discussed.

\subsection{Finite time velocity estimation}

Assume that $\ddot{x}, \dot{x}$ and $x$ are unbiased periodic functions with period $T_{f}$. In fact, if $\dot{x}$ or $\ddot{x}$ have a bias, then $x$ would not be periodical and bounded. Moreover, a constant bias in position cannot be identified from acceleration or velocity measurements because none of them will reflect such a bias. Now, consider the equations (4) and (5) evaluated at $T_{f}$

$$
\begin{aligned}
& \Psi^{<1>}\left(T_{f}\right)=\dot{x}\left(T_{f}\right)-\dot{x}(0)+\eta^{<1>}, \\
& \Psi^{<2>}\left(T_{f}\right)=x\left(T_{f}\right)-x(0)-\dot{x}(0) \cdot T_{f}+\eta^{<2>},
\end{aligned}
$$

where $x\left(T_{f}\right)=x(0)$ and $\dot{x}\left(T_{f}\right)=\dot{x}(0)$. Hence, an estimation $\hat{\dot{x}}(0)$ of $\dot{x}(0)$ can be obtained at $t=T_{f}$ :

$$
\begin{aligned}
\hat{\dot{x}}(0) & =-\frac{\Psi^{<2>}\left(T_{f}\right)}{T_{f}} \\
& =\dot{x}(0)-\frac{\eta^{<2>}}{T_{f}} .
\end{aligned}
$$

Then, if period is known, an estimation of velocity can be derived using equations (10) and (13):

$$
\hat{\dot{x}}(t)=\Psi^{<1>}(t)-\hat{\dot{x}}(0) .
$$

Next, the same approach can be repeated for position estimation.

\subsection{Importance of period knowledge}

Note that $x(t)=x(0)$ may hold for different time instants, but the correct estimation of velocity can only be ensured for $t=\kappa T_{f}$ with $\kappa \in \mathbb{N}$. It implies that estimation of the initial condition, $\dot{x}(0)$, without knowledge of the period may lead to big errors. To illustrate this idea, consider the next expression for acceleration

$$
\ddot{x}=\sin (1.5 t)+\sin (4.2 t),
$$

which is plotted with its corresponding velocity and position signals in Fig. 1. Suppose that measurements start at $t=A$. Note that there is a subperiod, between times $A$ and $B$, for both acceleration and velocity. Given that only the acceleration is available, this zero mean subperiod can be wrongly considered as the real period causing the error term $(x(B)-x(A)) /(B-A)$ to be present in the estimation $\hat{\dot{x}}(0)$, see Eq. (13). In next section, a solution to the problem of period estimation is presented.

\section{PERIOD IDENTIFICATION}

The algorithm for period estimation proposed in this paper is based on the features of the phase portrait $(\dot{x}, \ddot{x})$. To introduce the idea, consider the phase portrait $(\dot{x}, \ddot{x})$ of (15) depicted in Fig. 2. It is clear that after one period the trajectories pass through each point at least once. Nevertheless, there are a number of points and furthermore trajectory segments, where the trajectory passes more than once over a cycle. If we can select a point which is reached only once over a whole period, then an estimation of the period can be obtained by detecting the time elapsed between two consecutive passings over such point. The question is how to properly select this point.

Recall that the real phase portrait is not available because there is no velocity measurement. From Equation (4) it can be seen that the velocity estimation, $\dot{x}$, is shifted 


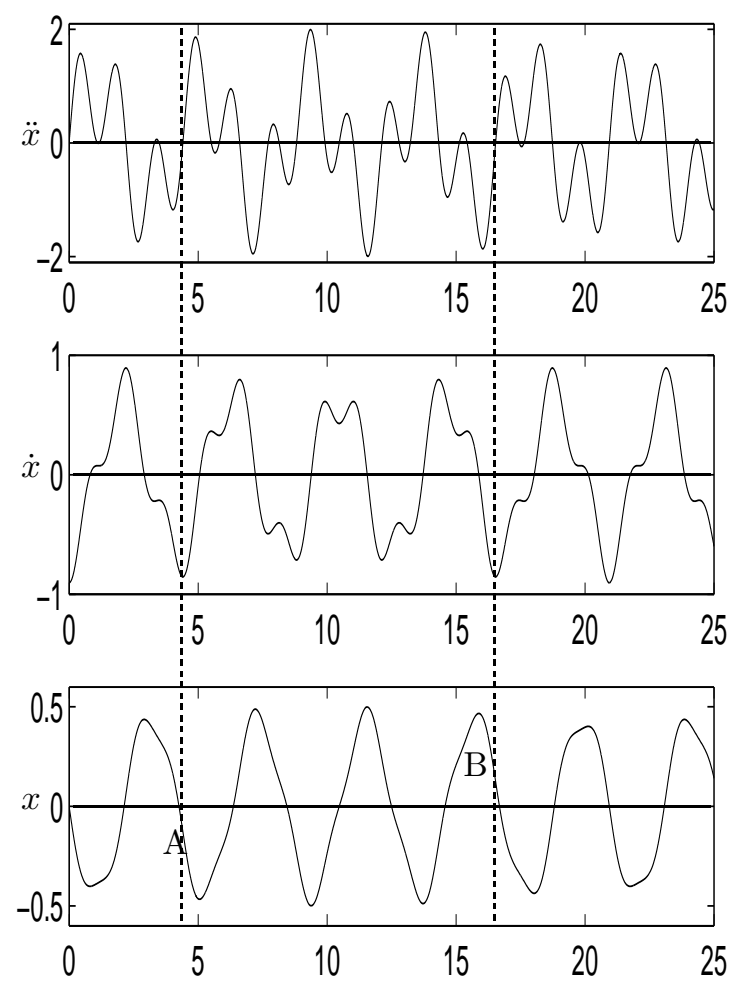

Fig. 1. Acceleration, Velocity and Position (sec).

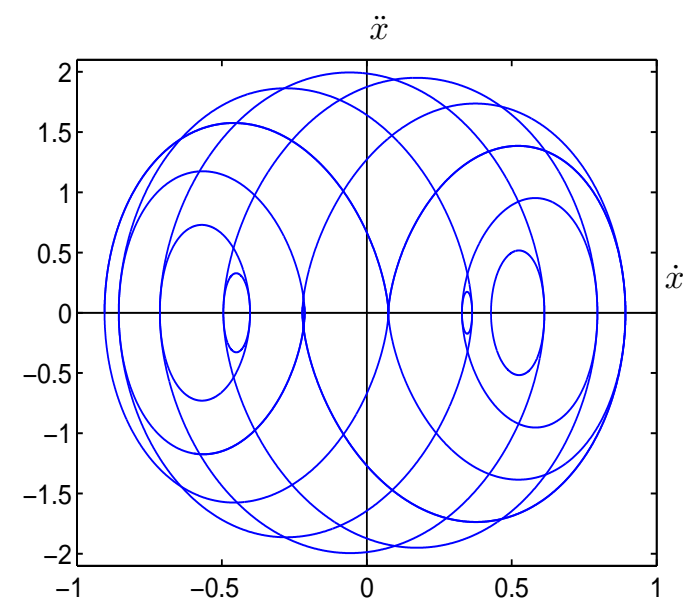

Fig. 2. Phase portrait $(\dot{x}, \ddot{x})$ for (15).

by its initial condition. It is possible to obtain a period estimation under the assumptions:

S1. $\dot{x}$ and $\ddot{x}$ are zero-mean periodical signals with unknown period $T_{f}$.

S2. On the period $T_{f}, \dot{x}$ has a unique global extremum, either a maximum or a minimum, represented by $\dot{x}_{M}$.

S3. On the period $T_{f}$, the time integrals $\eta^{<i>}, i=1,2$, are bounded by constants $\delta_{\eta<i>}$, i.e., $\left|\eta^{<i>}(t)\right|<$ $\delta_{\eta}<i>\forall t \in\left[0, T_{f}\right]$.

Remark 1. The Assumption S2 restricts the function $\dot{x}$ to have only a single occurrence of the respective global extremum over one period. Moreover, it implies that the magnitude of this global extremum is different from the magnitude of any other local extremum.

\subsection{Period Identification Algorithm}

Recall that $\dot{x}_{M}$ denotes the global extremum in velocity. Acceleration is in general a noisy measurement and the accuracy of integration is additionally limited by the sampling time. The algorithm for period estimation should perform the following tasks

(1) To ensure the detection of every occurrence, once each period, of the extremum $\dot{x}_{M}$. Due to the integration error, $\delta_{\eta<1>}$, detected extremum cannot be separated from the real $\dot{x}_{M}$ by a distance larger than $\delta_{\eta^{<1>}}$. The algorithm should ensure that the above mentioned

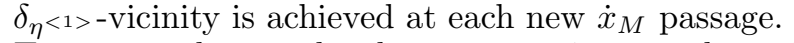

(2) To ensure that no local extremum is wrongly estimated as $\dot{x}_{M}$. In addition to possible extrema outside

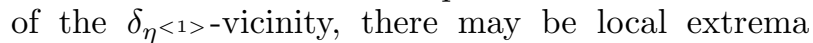
inside such a vicinity. These local extrema should be disregarded. The above implies that it is necessary

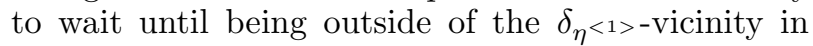
order to decide that a new detected extremum will be considered as a new occurrence of $\dot{x}_{M}$.

(3) In order to avoid accumulation of drift, the integral of acceleration, on which the detection of $\dot{x}_{M}$ is based, should be restarted once each period. This restart can be performed after a new $\dot{x}_{M}$ occurrence has been confirmed.

If the above conditions are fulfilled then an estimation of the period can be made as the difference between two consecutive detected occurrences of $\dot{x}_{M}$. Some definitions are introduced prior to present the algorithm. $\alpha$ is a parameter to be selected as 1 or -1 in order to detect a global maximum, or a global minimum respectively. $\dot{x}_{M-}$ is the extremum, different from $\dot{x}_{M}$, whose amplitude is the closest to $\dot{x}_{M}$. The notation $\Psi^{<1>}\left(t_{a}, t_{b}\right)$ indicates the integral of $\Psi$ from $t=t_{a}$ to $t=t_{b}$. Next we present the proposed period detection algorithm:

\section{Algorithm A1.}

Initialization of variables

$$
\begin{aligned}
& \alpha= \pm 1, t_{0}=0, t_{1}=0, f l=0, \\
& \delta>\delta_{\eta^{<1>}} \\
& \Psi_{m}^{<1>}=0,
\end{aligned}
$$

$$
\begin{aligned}
& \text { C0. If } \alpha \Psi^{<1>}\left(t_{0}, t\right)>\alpha \Psi_{m}^{<1>}-\delta f l \underline{\text { then }} \\
& \begin{aligned}
\Psi_{m}^{<1>} & =\Psi^{<1>}\left(t_{0}, t\right), \\
t_{1} & =t, \\
f l & =0 .
\end{aligned}
\end{aligned}
$$

C1. If $\alpha \Psi^{<1>}\left(t_{1}, t\right)<-2 \delta$ and $f l=0$ then

$$
\begin{aligned}
\Psi_{m}^{<1>} & =0, \\
\hat{T}_{f} & =t_{1}-t_{0}, \\
t_{0} & =t_{1}, \\
f l & =1,
\end{aligned}
$$


Remark 2. The only parameter to be tuned is $\delta$ whose value will be related to noise amplitude and is further discussed in the sequel.

We state the claims:

Lemma 1. Each occurrence of $\dot{x}_{M}$ is followed by only one fulfillment of condition $\mathbf{C 1}$ with a value for $t_{1}$ such that

$$
\dot{x}\left(t_{1}\right)>\dot{x}_{M}-\alpha \delta_{\eta<1>} .
$$

Proposition 2. If assumptions S1-S3 are fulfilled then, an estimation of the real period, $T_{f}$, is obtained by means of the algorithm A1 provided that

$$
\frac{1}{2}\left|\dot{x}_{M}-\dot{x}_{M^{-}}\right|>\delta>\delta_{\eta^{<1>}} .
$$

Assume that $\alpha=1$ is selected. Then, the algorithm A1 proceeds in the following manner:

- The condition $\mathbf{C 0}$ at the beginning is $\alpha \Psi^{<1>}(0, t)>$ 0 , and it is fulfilled for any positive quantity of the integral $\alpha \Psi^{<1>}(0, t)$.

- After the first fulfillment of $\mathbf{C 0}$ the assigment $\Psi_{m}^{<1>}=$ $\Psi^{<1>}(0, t)$ is done in order to save the new maximum, and the time of its occurrence is saved on $t_{1}$.

- The condition $\mathbf{C 1}$ is checked for the integral $\Psi^{<1>}\left(t_{1}, t\right)$, which is restarted at the instant $t_{1}$ of the last maximum detection of $\Psi^{<1>}\left(t_{0}, t\right)$. This condition is satisfied if $\Psi^{<1>}\left(t_{1}, t\right)$ becomes less than $-2 \delta$ and $f l=0$.

- The condition C1 serves for the detection purpose of a passage of the global maximum $\dot{x}_{M}$ (in order to distinguish it from $\dot{x}_{M-}$ or other local extremums). It is shown in the proof of Lemma 1, that after the first occurrence of $\dot{x}_{M}$ the condition (18) is fulfilled.

- Finally, note that when condition C1 is fulfilled, the integral $\Psi^{<1>}\left(t_{0}, t\right)$ contains the integrated acceleration with the initial time corresponding to the last maximum. Additionally, the condition $\mathbf{C O}$ takes the form $\Psi^{<1>}\left(t_{0}, t\right)>-\delta$. This change in the form of condition $\mathbf{C 0}$ is needed in order to take into account a possible drift due to the integration error $\delta_{\eta<1>}<\delta$.

\section{Proofs.}

Let the unique absolute extremum for $\dot{x}$ be a global maximum denoted as $\dot{x}_{M}$ (the proof in the case of a global minimum follows the same rationale). For this case $\alpha=1$ is selected and the conditions $\mathbf{C 0}$ and $\mathbf{C 1}$ are rewritten as

$$
\begin{array}{ll}
\Psi^{<1>}\left(t_{0}, t\right)>\Psi_{m}^{<1>} & \text { if } f l=0 \\
\Psi^{<1>}\left(t_{0}, t\right)>-\delta & \text { if } f l=1
\end{array}
$$

and

$$
\begin{aligned}
\Psi^{<1>}\left(t_{1}, t\right) & <-2 \delta \\
f l & =0,
\end{aligned}
$$

respectively. Note that condition C0 can take two forms, (20a) for $f l=0$ and (20b) for $f l=1$.

Proof of Lemma 1. This proof is divided into two parts:

- First, let us show that the condition

$$
\dot{x}\left(t_{1}\right) \in\left[\dot{x}_{M}-\delta_{\eta^{<1>}}, \dot{x}_{M}\right] .
$$

is satisfied independently on the initial conditions $\ddot{x}_{\circ}=\ddot{x}(0), \dot{x}_{\circ}=\dot{x}(0)$ and $x_{\circ}=x(0)$.

Define $T_{M}^{1}$ as the time of first appearance of $\dot{x}_{M}$ and suppose that the initial condition $\dot{x}_{\circ}$ is such that

$$
\dot{x}_{\circ}<\dot{x}_{M}-\delta_{\eta<1>},
$$

in the contrary case, (22) will be necessarily satisfied after the first fulfillment of $\mathbf{C 1}$. Then, considering (23), if (21) is not fulfilled between $t=0$ and $t=T_{M}^{1}$ we have

$$
\begin{aligned}
\Psi^{<1>}\left(t_{0}, T_{M}^{1}\right) & =\Psi^{<1>}\left(0, T_{M}^{1}\right) \\
& =\dot{x}_{M}-\dot{x}_{\circ}+\eta^{<1>} \\
& >\dot{x}_{M}-\dot{x}_{M}+\delta_{\eta^{<1>}}-\delta_{\eta^{<1>}} \\
& >0 .
\end{aligned}
$$

Thereby, C0, that is (20a), will be necessarily true at least for $\dot{x}(t)=\dot{x}_{M}$, recall that the errors are comprised in $\delta_{\eta<1>}$. The above implies that the correspondent time $t_{1}$ captured due to $\mathbf{C 0}$ is such that (22) is verified.

On the other hand, suppose that C1, see (21), is true before passing through $\dot{x}_{M}$. This implies that $\Psi^{<1>}\left(t_{0}, t\right)$ has decreased $2 \delta$ in magnitude since its last largest value. The assignments $\Psi_{m}^{<1>}=0$ and $f l=1$ due to $\mathbf{C 1}$ implies that condition $\mathbf{C 0}$ takes the form (20b). Now, note that the latter must be fulfilled before a new true event of $\mathbf{C 1}$. Since $t_{0}$ is reset to the time of occurrence of the last largest value of $\Psi^{<1>}\left(t_{0}, t\right)$, one obtains that

$$
\Psi^{<1>}\left(t_{0}, t\right)<2 \delta
$$

holds, after the assignments in $\mathbf{C 1}$ with $x_{M}$ still to appear. Then, there exist a time $t^{*}$ such that

$$
\dot{x}\left(t^{*}\right)=\dot{x}_{\circ} ; \quad \text { with } t^{*}<T_{f}
$$

and for $t=t^{*}$ one obtains

$$
\begin{aligned}
\Psi^{<1>}\left(t_{0}, t^{*}\right) & =\dot{x}\left(t^{*}\right)-\dot{x}\left(t_{0}\right)+\eta^{<1>} \\
& \geq-\delta_{\eta^{<1>}}>-\delta .
\end{aligned}
$$

Then, (20b) is fulfilled and C0 takes the form (20a) again. From the above, if $\dot{x}\left(t^{*}\right)=\dot{x}\left(t_{0}\right)<\dot{x}_{M}-\delta_{\eta^{<1>}}$ then condition (20) will be true at some time satisfying $\dot{x}(t) \in\left[\dot{x}_{M}-\delta_{\eta<1>}, \dot{x}_{M}\right]$.

This concludes the part that ensures that $t_{1}$ will be such that (22) holds independently of the initial conditions.

- Once $t_{1}$ satisfies (22), condition C1 is fulfilled only once after each $\dot{x}_{M}$ occurence. Consider that $\mathbf{C 1}$ is true after $(22)$ is satisfied then,

$$
\dot{x}\left(t_{0}\right) \in\left[\dot{x}_{M}-\delta_{\eta<1>}, \dot{x}_{M}\right],
$$

and note that $\mathbf{C 0}$ must be before $\mathbf{C 1}$ can be satisfied again. Next we show that $\mathbf{C 0}$, which is now in the form (20b), can only be true again in the vicinity $\dot{x}(t) \in\left[\dot{x}_{M}-\delta_{\eta^{<1>}}, \dot{x}_{M}\right]$. Recall that the local extremum closest in magnitude to $\dot{x}_{M}$ is $\dot{x}_{M}^{-}$. Then, for $\dot{x}(t)=\dot{x}_{M}^{-}$, after (24) holds, we obtain

$$
\begin{aligned}
& \Psi^{<1>}\left(t_{0}, t\right)=\dot{x}_{M}^{-}-\dot{x}\left(t_{0}\right)+\eta^{<1>} \\
& \Psi^{<1>}\left(t_{0}, t\right) \leq \dot{x}_{M}^{-}-\dot{x}_{M}+\eta^{<1>} .
\end{aligned}
$$

From (19) we have that $\dot{x}_{M}^{-}-\dot{x}_{M}<-2 \delta$, hence

$$
\begin{aligned}
& \Psi^{<1>}\left(t_{0}, t\right)<-2 \delta+\delta_{\eta^{<1>}} \\
& \Psi^{<1>}\left(t_{0}, t\right)<-\delta
\end{aligned}
$$

thus, $\dot{x}(t)$ must be larger than $\dot{x}_{M}^{-}$in order to fulfill again C0. This can only occur in vicinity of $\left[\dot{x}_{M}-\right.$ $\left.\delta_{\eta^{<1>}}, \dot{x}_{M}\right]$. On the other hand, $\mathbf{C 0}$ will necessarily be true in the latter vicinity, to show this consider the case $\dot{x}(t)=\dot{x}_{M}$ : 


$$
\begin{aligned}
& \Psi^{<1>}\left(t_{0}, t\right)=\dot{x}(t)-\dot{x}\left(t_{0}\right)+\eta^{<1>} \\
& \Psi^{<1>}\left(t_{0}, t\right) \in \dot{x}_{M}-\dot{x}_{M}-\left[-\delta_{\eta^{<1>}}, 0\right]+\eta^{<1>} \\
& \Psi^{<1>}\left(t_{0}, t\right)>-\delta_{\eta^{<1>}}>-\delta .
\end{aligned}
$$

Then, condition $\mathbf{C 0}$ is fulfilled and a new time $t_{1}$ will be captured such that (22) is satisfied. This concludes the proof of Lemma 1.

Proof of Proposition 2. Proposition 2 is a straightforward result of Lemma 1 . Note that if $(22)$ is satisfied, then for each subsequent $\mathbf{C 1}$ verification, the variables $t_{0}$ and $t_{1}$ will contain the time of detection of two consecutive global maxima of $\Psi^{<1>}$, with an error which depends on the noise and the sampling, $\delta_{\eta<1>}$. The condition $(22)$ cannot be ensured before a whole period has elapsed, then at least two periods are needed in order to ensure that a correct estimation of $T_{f}$ is obtained. Moreover, as the condition $\mathbf{C 1}$ is needed to compute the estimation, the first correct estimation may occur during the third period after launching the algorithm, when $\Psi^{<1>}$ exits the $2 \delta$ vicinity.

It is worth to stress that the developed algorithm does not impose a restriction on possible values for the amplitude and the period of the signals. Let us remark that the accuracy does not depends on the parameter $\delta$ of the algorithm, the error in the estimation is directly related to $\delta_{\eta<1>}$ which is inherent to the system.

\section{VELOCITY AND POSITION ESTIMATION BASED ON PERIOD ESTIMATION}

The period estimation, $\hat{T}_{f}$, obtained in the previous section can be used to estimate $\dot{x}_{M}$ and in turn to obtain a real time velocity estimation. The fact that $\dot{x}_{M}$ is estimated at each period will be used in order to compensate the drift due to errors previously mentioned. Define $T_{M}^{i}$ the time of $i$-th occurrence of the extremum $\dot{x}_{M}$, and denote $\hat{T}_{M}^{i}$ its estimate calculated after fulfillment of condition $\mathbf{C 1}$, then $\hat{T}_{M}^{i}=t_{1}$. Let $\hat{T}_{f}^{i}$ be the period estimation corresponding to the time $T_{M}^{i}$, which is obtained on the $i$-th iteration of Algorithm A1, then $\hat{T}_{f}^{i}=\hat{T}_{M}^{i}-\hat{T}_{M}^{i-1}$. Thus, based on (13) we obtain

$$
\hat{\dot{x}}\left(T_{M}^{i}\right)=-\frac{\Psi^{<2>}\left(\hat{T}_{M}^{i-1}, \hat{T}_{M}^{i}\right)}{\left(\hat{T}_{f}^{i}\right)} .
$$

Analogously, an estimation $\hat{x}\left(T_{M}^{i}\right)$ can be derived by using the estimated period $\hat{T}_{f}^{i}$ and the third integral of $\Psi$ that is

$$
\begin{aligned}
\Psi^{<3>}\left(T_{M}^{i-1}, T_{M}^{i}\right)= & x^{<1>}\left(T_{M}^{i}\right)-x^{<1>}\left(T_{M}^{i-1}\right) \\
& -x\left(T_{M}^{i-1}\right)\left(T_{M}^{i}-T_{M}^{i-1}\right) \\
& -\frac{1}{2} \dot{x}\left(T_{M}^{i-1}\right)\left(T_{M}^{i}-T_{M}^{i-1}\right)^{2} \\
& +\eta^{<3>}\left(T_{M}^{i-1}, T_{M}^{i}\right),
\end{aligned}
$$

then, assuming that $x^{<1>}\left(T_{M}^{i}\right)=x^{<1>}\left(T_{M}^{i-1}\right)$,

$$
\hat{x}\left(\hat{T}_{M}^{i}\right)=-\frac{\Psi^{<3>}\left(\hat{T}_{M}^{i-1}, \hat{T}_{M}^{i}\right)}{\hat{T}_{f}}+\frac{1}{2} \hat{\dot{x}}\left(\hat{T}_{M}^{i-1}\right) \hat{T}_{f} .
$$

In the ideal case of zero noise, exact integration and exact detection of the extremum instants $T_{M}^{i}$ 's, we have that $\Psi^{<1>}\left(\hat{T}_{M}^{i-1}, \hat{T}_{M}^{i}\right)=0\left(\delta_{\eta<1>}=0\right)$. Although, in general $\Psi^{<1>}\left(\hat{T}_{M}^{i-1}, \hat{T}_{M}^{i}\right) \neq 0$ and the assumption $\dot{x}\left(\hat{T}_{M}^{i-1}\right)=$
$\dot{x}\left(\hat{T}_{M}^{i}\right)$ is made in order to obtain the period estimation $\hat{T}_{f}^{i}$. An estimation of the error $\eta^{<1>}$ can be obtained as

$$
\hat{\eta}^{<1>}\left(\hat{T}_{M}^{i}\right)=\Psi^{<1>}\left(\hat{T}_{M}^{i-1}, \hat{T}_{M}^{i}\right)
$$

Using (28) we can obtain an estimation of the errors $\eta^{<2>}$ and $\eta^{<3>}$ allowing to compensate them in $\Psi^{<2>}$ and $\Psi^{<3>}$, before computing $\hat{\dot{x}}\left(\hat{T}_{M}^{(i)}\right)$ and $\hat{x}\left(\hat{T}_{M}^{(i)}\right)$ respectively. In order to do that, we consider $\hat{\eta}^{<1>}\left(T_{M}^{i}\right)$ as the result of the integration of the constant magnitude $\frac{\hat{\eta}^{<1>}\left(\hat{T}_{M}^{i}\right)}{\hat{T}_{f}^{i}}$ over the interval $\left[\hat{T}_{M}^{i-1}, \hat{T}_{M}^{i}\right]$. Thereby, the next error estimations are obtained

$$
\begin{aligned}
& \hat{\eta}^{<2>}\left(\hat{T}_{M}^{i}\right)=\frac{1}{2} \Psi^{<1>}\left(\hat{T}_{M}^{i-1}, \hat{T}_{M}^{i}\right) \hat{T}_{f} \\
& \hat{\eta}^{<3>}\left(\hat{T}_{M}^{i}\right)=\frac{1}{6} \Psi^{<1>}\left(\hat{T}_{M}^{i-1}, \hat{T}_{M}^{i}\right) \hat{T}_{f}^{2} .
\end{aligned}
$$

Introducing in (25) and (27) the above errors yields

$$
\begin{aligned}
\hat{\dot{x}}\left(\hat{T}_{M}^{i}\right)= & -\frac{\Psi^{<2>}\left(\hat{T}_{M}^{i-1}, \hat{T}_{M}^{i}\right)}{\left(\hat{T}_{f}^{i}\right)}+\frac{1}{2} \Psi^{<1>}\left(\hat{T}_{M}^{i-1}, \hat{T}_{M}^{i}\right), \\
\hat{x}\left(\hat{T}_{M}^{i}\right)= & -\frac{\Psi^{<3>}\left(\hat{T}_{M}^{i-1}, \hat{T}_{M}^{i}\right)}{\hat{T}_{f}} \\
& -\left[\frac{1}{2} \hat{\dot{x}}\left(\hat{T}_{M}^{i-1}\right)-\frac{1}{6} \Psi^{<1>}\left(\hat{T}_{M}^{i-1}, \hat{T}_{M}^{i}\right)\right] \hat{T}_{f} .
\end{aligned}
$$

Finally, the estimation of velocity and position is obtained through the next expressions

$$
\begin{aligned}
& \hat{\dot{x}}(t)=\Psi^{<1>}\left(\hat{T}_{M}^{i}, t\right)-\hat{\dot{x}}\left(\hat{T}_{M}^{i}\right), \\
& \hat{x}(t)=\Psi^{<2>}\left(\hat{T}_{M}^{i}, t\right)-\hat{x}\left(\hat{T}_{M}^{i}\right) .
\end{aligned}
$$

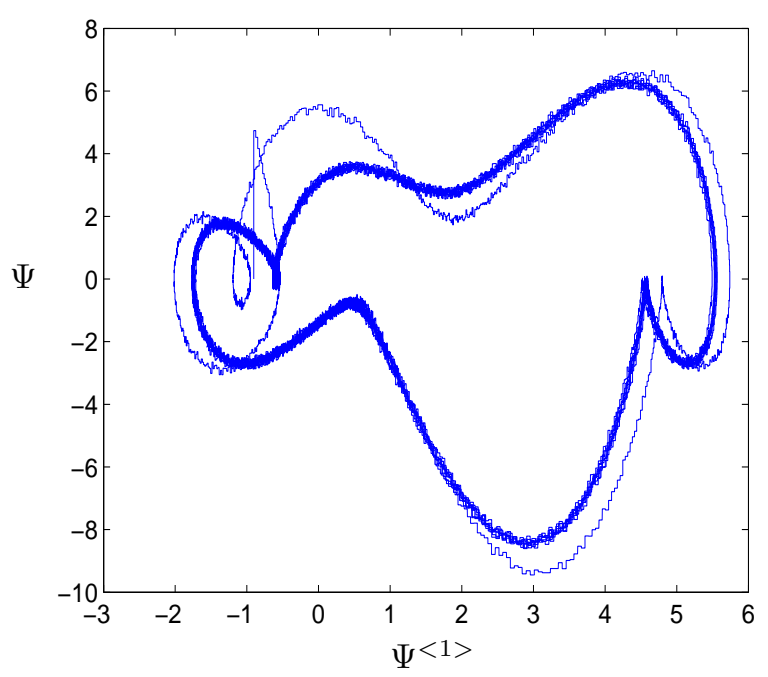

Fig. 3. Phase portrait $\left(\Psi^{<1>}, \Psi\right)$ for system (35).

\section{EXAMPLE}

Expressions (33) and (34) (with the results obtained from algorithm A1) were applied for velocity and position estimation with acceleration measurements for a variant of Van der Pol oscillator

$$
\begin{aligned}
& \ddot{x}+0.1(x+1)(x-1)(x-2.2) \dot{x}+x=0 \\
& \Psi(t)=\ddot{x}(t)+\eta(t)
\end{aligned}
$$


The parameters in (35) were proposed in Karreman and Prood (1995) as a mathematical model for an isometric contraction of the mammalian cardiac muscle. The zero mean noise has been selected to satisfy $|\eta(t)|<0.2$. The phase portrait $(\ddot{x}, \dot{x})$ of $(35)$, with initial conditions $x_{0}=3$ and $\dot{x}_{0}=-2$, is displayed in Fig. 3. The estimation results are shown in Fig. 4.
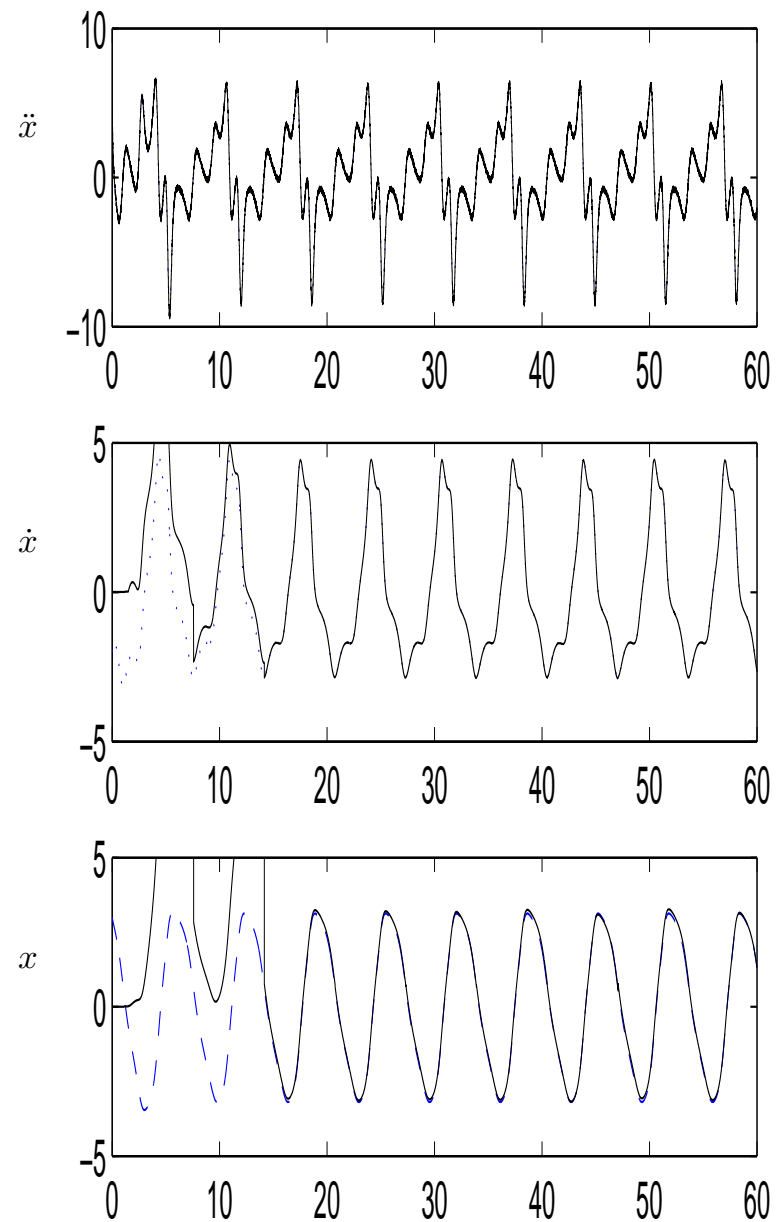

Fig. 4. Velocity and position estimation for (35).

\section{CONCLUSIONS}

For a class of oscillatory motion, a scheme for velocity and position estimation based on noisy acceleration measurements is presented. The inherent error accumulation, due to integration drift, is compensated using information about the period of oscillation. An algorithm for estimation of the aforementioned period of oscillation is proposed. No model of the motion or a priori information on the period of the signals are required. As a result, the proposed scheme is suitable as a standalone solution for estimation of position and velocity using only inertial information without any aiding measurements. Further research is directed to the application of the proposed solution on a practical scenario with real measurement data.

\section{REFERENCES}

Bernardin, S.L. (2006). Wavelet processing for pitch period estimation. In Proceedings of the 38th Southeastern Symposium on System Theory. Cookeville, TN, USA.

Feder, M. (1993). Parameter estimation and extraction of helicopter signals oberved with a wide-band interference. IEEE Transactions on Signal Processing, 41(1), $232-244$.

Grewal, M.S., Weill, L.R., and Andrew, A. (2007). Global Positioning, Inertial Navigation and Integration. John Wiley \& Sons, 2nd edition.

Guangyu, K. and Shize, G. (2009). Improving AMDF for pitch period detection. In International Conference on Electronic Measurement and Instruments.

Karreman, G. and Prood, C. (1995). Heart muscle contraction oscillation. International Journal of Bio-Medical Computing, 38, 49-53.

Liu, T., Inoue, Y., and shibata, K. (2009). Development of a wearable sensor system for quantitative gait analysis. Measurement, 42, 978-988.

Min, Y., Yingchun, Y., and Zhaohui, W. (2005). A pitched-based rapid speech segmentation for speaker indexing. In Seventh IEEE International Symposium on Multimedia.

Peeters, G. (2006). Music pitch representation by periodicity measures based on combined temporal and spectral representations. In ICASSP 2006 Proceedings. IEEE International conference on Acoustics, Speech and Signal Processing, volume 5, V.

Rogers, R.M. (2003). Applied Mathematics in Integrated Navigation Systems. Reston : American Institute of Aeronautics and Astronautics, Inc.

Schepers, H.M., van Asseldonk, E.H., Baten, C.T., and Veltnik, P.H. (2010). Ambulatory estimation of foot placement during walking using inertial sensors. Journal of biomechanics, 43, 3138-3143.

Spence, G. and Clarke, I. (2000). A time-domain analysis of multiple periodic signals. In IEE Seminar on Timescale and Time-frequency Analysis and Applications, 2/1-2/10. London.

Thong, Y., Woolfson, M., Crowe, J., Hayes-Gill, B., and Jones, D. (2004). Numerical double integration of acceleration measurements in noise. Measurement, 36(1), 73 - 92. doi:10.1016/j.measurement.2004.04.005. 